\title{
Bartholdi Zeta Functions for Hypergraphs
}

\author{
Iwao SATO \\ Oyama National College of Technology, \\ Oyama, Tochigi 323-0806, JAPAN \\ e-mail: isato@oyama-ct.ac.jp
}

Submitted: Oct 22, 2006; Accepted: Dec 19, 2006; Published: Jan 3, 2007

Mathematical Subject Classification: 05C50, 15A15

\begin{abstract}
Recently, Storm [8] defined the Ihara-Selberg zeta function of a hypergraph, and gave two determinant expressions of it. We define the Bartholdi zeta function of a hypergraph, and present a determinant expression of it. Furthermore, we give a determinant expression for the Bartholdi zeta function of semiregular bipartite graph. As a corollary, we obtain a decomposition formula for the Bartholdi zeta function of some regular hypergraph.
\end{abstract}

\section{Introduction}

Graphs and digraphs treated here are finite. Let $G$ be a connected graph and $D$ the symmetric digraph corresponding to $G$. Set $D(G)=\{(u, v),(v, u) \mid u v \in E(G)\}$. For $e=(u, v) \in D(G)$, set $u=o(e)$ and $v=t(e)$. Furthermore, let $e^{-1}=(v, u)$ be the inverse of $e=(u, v)$.

A path $P$ of length $n$ in $D$ (or $G$ ) is a sequence $P=\left(e_{1}, \cdots, e_{n}\right)$ of $n$ arcs such that $e_{i} \in D(G), t\left(e_{i}\right)=o\left(e_{i+1}\right)(1 \leq i \leq n-1)$. If $e_{i}=\left(v_{i-1}, v_{i}\right)$ for $i=1, \cdots, n$, then we write $P=\left(v_{0}, v_{1}, \cdots, v_{n-1}, v_{n}\right)$. Set $|P|=n, o(P)=o\left(e_{1}\right)$ and $t(P)=t\left(e_{n}\right)$. Also, $P$ is called an $(o(P), t(P))$-path. We say that a path $P=\left(e_{1}, \cdots, e_{n}\right)$ has a backtracking or a bump at $t\left(e_{i}\right)$ if $e_{i+1}^{-1}=e_{i}$ for some $i(1 \leq i \leq n-1)$. A $(v, w)$-path is called a $v$-cycle (or $v$-closed path) if $v=w$. The inverse cycle of a cycle $C=\left(e_{1}, \cdots, e_{n}\right)$ is the cycle $C^{-1}=\left(e_{n}^{-1}, \cdots, e_{1}^{-1}\right)$.

We introduce an equivalence relation between cycles. Two cycles $C_{1}=\left(e_{1}, \cdots, e_{m}\right)$ and $C_{2}=\left(f_{1}, \cdots, f_{m}\right)$ are called equivalent if $f_{j}=e_{j+k}$ for all $j$. The inverse cycle of $C$ is not equivalent to $C$. Let $[C]$ be the equivalence class which contains a cycle $C$. Let $B^{r}$ be the cycle obtained by going $r$ times around a cycle $B$. Such a cycle is called a multiple of $B$. A cycle $C$ is reduced if both $C$ and $C^{2}$ have no backtracking. Furthermore, a cycle $C$ is prime if it is not a multiple of a strictly smaller cycle. Note that each equivalence 
class of prime, reduced cycles of a graph $G$ corresponds to a unique conjugacy class of the fundamental group $\pi_{1}(G, v)$ of $G$ at a vertex $v$ of $G$.

Let $G$ be a connected graph. Then the cyclic bump count $\operatorname{cbc}(\pi)$ of a cycle $\pi=$ $\left(\pi_{1}, \cdots, \pi_{n}\right)$ is

$$
c b c(\pi)=\left|\left\{i=1, \cdots, n \mid \pi_{i}=\pi_{i+1}^{-1}\right\}\right|,
$$

where $\pi_{n+1}=\pi_{1}$.

Bartholdi [1] introduced the Bartholdi zeta function of a graph. The Bartholdi zeta function of $G$ is defined by

$$
\zeta(G, u, t)=\prod_{[C]}\left(1-u^{c b c(C)} t^{|C|}\right)^{-1}
$$

where $[C]$ runs over all equivalence classes of prime cycles of $G$, and $u, t$ are complex variables with $|u|,|t|$ sufficiently small.

If $u=0$, then, since $0^{0}=1$, the Bartholdi zeta function of $G$ is the (Ihara) zeta function of $G$ (see [5]):

$$
\zeta(G, 0, t)=\mathbf{Z}(G, t)=\prod_{[C]}\left(1-t^{|C|}\right)^{-1}
$$

where $[C]$ runs over all equivalence classes of prime, reduced cycles of $G$. Ihara [5] defined zeta functions of graphs, and showed that the reciprocals of zeta functions of regular graphs are explicit polynomials. A zeta function of a regular graph $G$ associated with a unitary representation of the fundamental group of $G$ was developed by Sunada $[9,10]$. Hashimoto [4] treated multivariable zeta functions of bipartite graphs. Bass [2] generalized Ihara's result on the zeta function of a regular graph to an irregular graph $G$, and showed that the reciprocal of the zeta function of $G$ is given by

$$
\mathbf{Z}(G, t)^{-1}=\left(1-t^{2}\right)^{r-1} \operatorname{det}\left(\mathbf{I}-t \mathbf{A}(G)+t^{2}\left(\mathbf{D}_{G}-\mathbf{I}\right)\right)
$$

where $r$ is the Betti number of $G$, and $\mathbf{D}_{G}=\left(d_{i j}\right)$ is the diagonal matrix with $d_{i i}=$ $\operatorname{deg}_{G} v_{i}\left(V(G)=\left\{v_{1}, \cdots, v_{n}\right\}\right)$. Stark and Terras [7] gave an elementary proof of this formula, and discussed three different zeta functions of any graph. Various proofs of Bass' Theorem were given by Kotani and Sunada [6], and Foata and Zeilberger [3].

Bartholdi [1] gave a determinant expression of the Bartholdi zeta function of a graph.

Theorem 1 (Bartholdi) Let $G$ be a connected graph with $n$ vertices and $m$ unoriented edges. Then the reciprocal of the Bartholdi zeta function of $G$ is given by

$$
\zeta(G, u, t)^{-1}=\left(1-(1-u)^{2} t^{2}\right)^{m-n} \operatorname{det}\left(\mathbf{I}-t \mathbf{A}(G)+(1-u)\left(\mathbf{D}_{G}-(1-u) \mathbf{I}\right) t^{2}\right) .
$$

Storm [8] defined the Ihara-Selberg zeta function of a hypergraph. A hypergraph $H=(V(H), E(H))$ is a pair of a set of hypervertices $V(H)$ and a set of hyperedges $E(H)$, which the union of all hyperedges is $V(H)$. A hypervertex $v$ is incident to a hyperedge $e$ if $v \in e$. For a hypergraph $H$, its dual $H^{*}$ is the hypergraph obtained by letting its hypervertex set be indexed by $E(H)$ and its hyperedge set by $V(H)$. 
A bipartite graph $B_{H}$ associated with a hypergraph $H$ is defined as follows: $V\left(B_{H}\right)=$ $V(H) \cup E(H)$ and $v \in V(H)$ and $e \in E(H)$ are adjacent in $B_{H}$ if $v$ is incident to $e$. Let $V(H)=\left\{v_{1}, \ldots, v_{n}\right\}$. Then an adjacency matrix $\mathbf{A}(H)$ of $H$ is defined as a mtarix whose rows and columns are parameterized by $V(H)$, and $(i, j)$-entry is the number of directed paths in $B_{H}$ from $v_{i}$ to $v_{j}$ of length 2 with no backtracking.

Let $H$ be a hypergraph. A path $P$ of length $n$ in $H$ is a sequence $P=\left(v_{1}, e_{1}, v_{2}, e_{2}, \cdots\right.$, $\left.e_{n}, v_{n+1}\right)$ of $n+1$ hypervertices and $n$ hyperedges such that $v_{i} \in V(H), e_{j} \in E(H), v_{1} \in e_{1}$, $v_{n+1} \in e_{n}$ and $v_{i} \in e_{i}, e_{i-1}$ for $i=2, \ldots, n-1$. Set $|P|=n, o(P)=v_{1}$ and $t(P)=v_{n+1}$. Also, $P$ is called an $(o(P), t(P))$-path. We say that a path $P$ has a hyperedge backtracking if there is a subsequence of $P$ of the form $(e, v, e)$, where $e \in E(H), v \in V(H)$. A $(v, w)$-path is called a $v$-cycle (or $v$-closed path) if $v=w$.

We introduce an equivalence relation between cycles. Such two cycles $C_{1}=\left(v_{1}, e_{1}, v_{2}\right.$, $\left.\cdots, e_{m}, v_{1}\right)$ and $C_{2}=\left(w_{1}, f_{1}, w_{2}, \cdots, f_{m}, w_{1}\right)$ are called equivalent if $w_{j}=v_{j+k}$ and $f_{j}=$ $e_{j+k}$ for all $j$. Let $[C]$ be the equivalence class which contains a cycle $C$. Let $B^{r}$ be the cycle obtained by going $r$ times around a cycle $B$. Such a cycle is called a multiple of $B$. A cycle $C$ is reduced if both $C$ and $C^{2}$ have no hyperedge backtracking. Furthermore, a cycle $C$ is prime if it is not a multiple of a strictly smaller cycle.

The Ihara-Selberg zeta function of $H$ is defined by

$$
\zeta_{H}(t)=\prod_{[C]}\left(1-t^{|C|}\right)^{-1},
$$

where $[C]$ runs over all equivalence classes of prime, reduced cycles of $H$, and $t$ is a complex variable with $|t|$ sufficiently small(see [8]).

Let $H$ be a hypergraph with $E(H)=\left\{e_{1}, \ldots, e_{m}\right\}$, and let $\left\{c_{1}, \ldots, c_{m}\right\}$ be a set of $m$ colors, where $c\left(e_{i}\right)=c_{i}$. Then an edge-colored graph $G H_{c}$ is defined as a graph with vertex set $V(H)$ and edge set $\{v w \mid v, w \in V(H) ; v, w \in e \in E(H)\}$, where an edge $v w$ is colored $c_{i}$ if $v, w \in e_{i}$.

Let $G H_{c}^{o}$ be the symmetric digraph corresponding to the edge-clored graph $G H_{c}$. Then the oriented line graph $H_{L}^{o}=\left(V_{L}, E_{L}^{o}\right)$ associated with $G H_{c}^{o}$ by

$$
\begin{aligned}
& V_{L}=D\left(G H_{c}^{o}\right), \text { and } E_{L}^{o}=\left\{\left(e_{i}, e_{j}\right) \in D\left(G H_{c}^{o}\right) \times D\left(G H_{c}^{o}\right) \mid c\left(e_{i}\right) \neq\right. \\
& \left.c\left(e_{j}\right), t\left(e_{i}\right)=o\left(e_{j}\right)\right\},
\end{aligned}
$$

where $c\left(e_{i}\right)$ is the color assigned to the oriented edge $e_{i} \in D\left(G H_{c}^{o}\right)$. The Perron-Frobenius operator $T: C\left(V_{L}\right) \longrightarrow C\left(V_{L}\right)$ is given by

$$
(T f)(x)=\sum_{e \in E_{o}(x)} f(t(e))
$$

where $E_{o}(x)=\left\{e \in E_{L}^{o} \mid o(e)=x\right\}$ is the set of all oriented edges with $x$ as their origin vertex, and $C\left(V_{L}\right)$ is the set of functions from $V_{L}$ to the complex number field $\mathbf{C}$.

Storm [8] gave two nice determinant expressions of the Ihara-Selberg zeta function of a hypergraph by using the results of Kotani and Sunada [6], and Bass [2]. 
Theorem 2 (Storm) Let $H$ be a finite, connected hypergraph such that every hypervetex is in at least two hyperedges. Then

$$
\zeta_{H}(t)^{-1}=\operatorname{det}(\mathbf{I}-t T)=(1-t)^{m-n} \operatorname{det}\left(\mathbf{I}-\sqrt{t} \mathbf{A}\left(B_{H}\right)+t \mathbf{Q}_{B_{H}}\right),
$$

where $n=\left|V\left(B_{H}\right)\right|, m=\left|E\left(B_{H}\right)\right|$ and $\mathbf{Q}_{B_{H}}=\mathbf{D}_{B_{H}}-\mathbf{I}$.

Furthermore, Storm [8] presented the Ihara-Selberg zeta function of a $(d, r)$-regular hypergraph by using the results of Hashimoto [4].

In Section 2, we define the Bartholdi zeta function of a hypergraph, and present a determinant expression of it. In Section 3, we give a decomposition formula (Theorem 4) for the Bartholdi zeta function of semiregular bipartite graph. As a corollary, we obtain a decomposition formula for the Bartholdi zeta function of some regular hypergraph. In Section 4, we prove Theorem 4 by using an analogue of Hashimoto's method [4].

\section{Bartholdi zeta function of a hypergraph}

Let $H$ be a hypergraph. Then a path $P=\left(v_{1}, e_{1}, v_{2}, e_{2}, \cdots, e_{n}, v_{n+1}\right)$ has a (broad) backtracking or (broad) bump at $e$ or $v$ if there is a subsequence of $P$ of the form $(e, v, e)$ or $(v, e, v)$, where $e \in E(H), v \in V(H)$. Furthermore, the cyclic bump count $\operatorname{cbc}(C)$ of a cycle $C=\left(v_{1}, e_{1}, v_{2}, e_{2}, \cdots, e_{n}, v_{1}\right)$ is

$$
c b c(C)=\left|\left\{i=1, \cdots, n \mid v_{i}=v_{i+1}\right\}\right|+\left|\left\{i=1, \cdots, n \mid e_{i}=e_{i+1}\right\}\right|,
$$

where $v_{n+1}=v_{1}$ and $e_{n+1}=e_{1}$.

The Bartholdi zeta function of $H$ is defined by

$$
\zeta(H, u, t)=\prod_{[C]}\left(1-u^{c b c(C)} t^{|C|}\right)^{-1}
$$

where $[C]$ runs over all equivalence classes of prime cycles of $H$, and $u, t$ are complex variables with $|u|,|t|$ sufficiently small.

If $u=0$, then the Bartholdi zeta function of $H$ is the Ihara-Selberg zeta function of $H$.

A determinant expression of the Bartholdi zeta function of a hypergraph is given as follows:

Theorem 3 Let $H$ be a finite, connected hypergraph such that every hypervetex is in at least two hyperedges. Then

$\zeta(H, u, t)=\zeta\left(B_{H}, u, \sqrt{t}\right)=\left(1-(1-u)^{2} t\right)^{-(m-n)} \operatorname{det}\left(\mathbf{I}-\sqrt{t} \mathbf{A}\left(B_{H}\right)+(1-u) t\left(\mathbf{D}_{B_{H}}-(1-u) \mathbf{I}\right)\right)^{-1}$ where $n=\left|V\left(B_{H}\right)\right|$ and $m=\left|E\left(B_{H}\right)\right|$. 
Proof. The argument is an analogue of Storm's method [8].

At first, we show that there exists a one-to-one correspondence between equivalence classes of prime cycles of length $l$ in $H$ and those of prime cycles of length $2 l$ in $B_{H}$, and $c b c(C)=c b c(\tilde{C})$ for any prime cycle $C$ in $H$ and the corresponding cycle $\tilde{C}$ in $B_{H}$.

Let $C=\left(v_{1}, e_{1}, v_{2}, \ldots, v_{l}, e_{l}, v_{1}\right)$ be a prime cycle of length $l$ in $H$. Then a cycle $\tilde{C}=\left(v_{1}, v_{1} e_{1}, e_{1}, \ldots, v_{l}, v_{l} e_{l}, e_{l}, e_{l} v_{1}, v_{1}\right)$ is a prime cycle of length $2 l$ in $B_{H}$. Thus, there exists a one-to-one correspondence between equivalence classes of prime cycles of length $l$ in $H$ and those of prime cycles of length $2 l$ in $B_{H}$.

Let $C$ a prime cycle in $H$ and $\tilde{C}$ a prime cycle corresponding to $C$ in $B_{H}$. Then there exists a subsequence $(v, e, v)$ (or $(e, v, e))$ in $C$ if and only if there exists a subsequence $(v, v e, e, e v, v)($ or $(e, e v, v, v e, e))$ in $\tilde{C}$. Thus, we have $c b c(C)=c b c(\tilde{C})$.

Therefore, it follows that

$$
\zeta(H, u, t)=\prod_{[C]}\left(1-u^{c b c(C)} t^{|C|}\right)^{-1}=\prod_{[\tilde{C}]}\left(1-u^{c b c(\tilde{C})} t^{|\tilde{C}| / 2}\right)^{-1}=\zeta\left(B_{H}, u, \sqrt{t}\right),
$$

where $[C]$ and $[\tilde{C}]$ runs over all equivalence classes of prime cycles in $H$ and $B_{H}$, respectively.

By Theorem 1, we have

$$
\zeta(H, u, t)=\left(1-(1-u)^{2} t\right)^{-(m-n)} \operatorname{det}\left(\mathbf{I}-\sqrt{t} \mathbf{A}\left(B_{H}\right)+(1-u) t\left(\mathbf{D}_{B_{H}}-(1-u) \mathbf{I}\right)\right)^{-1},
$$

where $n=|V(H)|$ and $m=|E(H)|$.

If $u=0$, then Theorem 3 implies Theorem 2 .

Corollary 1 Let $H$ be a finite, connected hypergraph such that every hypervetex is in at least two hyperedges. Then

$$
\zeta(H, u, t)=\zeta\left(H^{*}, u, t\right) .
$$

Proof. By the fact that $B_{H}=B_{H^{*}}$.

\section{Bartholdi zeta functions of $(d, r)$-regular hypergraphs}

At first, we state a decomposition formula for the Bartholdi zeta function of a semiregular bipartite graph. Hashimoto [4] presented a determinant expression for the Ihara zeta function of a semiregular bipartite graph. We generalize Hashimoto's result on the Thara zeta function to the Bartholdi zeta function.

A graph $G$ is called bipartite, denoted by $G=\left(V_{1}, V_{2}\right)$ if there exists a partition $V(G)=V_{1} \cup V_{2}$ of $V(G)$ such that the vertices in $V_{i}$ are mutually nonadjacent for $i=1,2$. A bipartite graph $G=\left(V_{1}, V_{2}\right)$ is called $\left(q_{1}+1, q_{2}+1\right)$-semiregular if $\operatorname{deg}_{G} v=q_{i}+1$ for each $v \in V_{i}(i=1,2)$. For a $\left(q_{1}+1, q_{2}+1\right)$-semiregular bipartite graph $G=\left(V_{1}, V_{2}\right)$, let $G^{[i]}$ be the graph with vertex set $V_{i}$ and edge set $\left\{P\right.$ : reduced path ||$\left.P \mid=2 ; o(P), t(P) \in V_{i}\right\}$ for $i=1,2$. Then $G^{[1]}$ is $\left(q_{1}+1\right) q_{2}$-regular, and $G^{[2]}$ is $\left(q_{2}+1\right) q_{1}$-regular.

A determinant expression for the Bartholdi zeta function of a semiregular bipartite graph is given as follows. For a graph $G$, let $\operatorname{Spec}(G)$ be the set of all eigenvalues of the adjacency matrix of $G$. 
Theorem 4 Let $G=\left(V_{1}, V_{2}\right)$ be a connected $\left(q_{1}+1, q_{2}+1\right)$-semiregular bipartite graph with $\nu$ vertices and $\epsilon$ edges. Set $\left|V_{1}\right|=n$ and $\left|V_{2}\right|=m(n \leq m)$. Then

$$
\begin{aligned}
& \zeta(G, u, t)^{-1}=\left(1-(1-u)^{2} t^{2}\right)^{\epsilon-\nu}\left(1+(1-u)\left(q_{2}+u\right) t^{2}\right)^{m-n} \\
\times & \prod_{j=1}^{n}\left(1-\left(\lambda_{j}^{2}-(1-u)\left(q_{1}+q_{2}+2 u\right)\right) t^{2}+(1-u)^{2}\left(q_{1}+u\right)\left(q_{2}+u\right) t^{4}\right) \\
= & \left(1-(1-u)^{2} t^{2}\right)^{\epsilon-\nu}\left(1+(1-u)\left(q_{2}+u\right) t^{2}\right)^{m-n} \operatorname{det}\left(\mathbf{I}_{n}-\left(\mathbf{A}^{[1]}-\left(\left(q_{2}-1\right)\right.\right.\right. \\
& \left.\left.\left.+\left(q_{1}+q_{2}-2\right) u+2 u^{2}\right) \mathbf{I}_{n}\right) t^{2}+(1-u)^{2}\left(q_{1}+u\right)\left(q_{2}+u\right) t^{4} \mathbf{I}_{n}\right) \\
= & \left(1-(1-u)^{2} t^{2}\right)^{\epsilon-\nu}\left(1+(1-u)\left(q_{1}+u\right) t^{2}\right)^{n-m} \operatorname{det}\left(\mathbf{I}_{m}-\left(\mathbf{A}^{[2]}-\left(\left(q_{1}-1\right)\right.\right.\right. \\
& \left.\left.\left.+\left(q_{1}+q_{2}-2\right) u+2 u^{2}\right) \mathbf{I}_{m}\right) t^{2}+(1-u)^{2}\left(q_{1}+u\right)\left(q_{2}+u\right) t^{4} \mathbf{I}_{m}\right),
\end{aligned}
$$

where $\operatorname{Spec}(G)=\left\{ \pm \lambda_{1}, \cdots, \pm \lambda_{n}, 0, \cdots, 0\right\}$ and $\mathbf{A}^{[i]}=\mathbf{A}\left(G^{[i]}\right)(i=1,2)$.

The proof of Theorem 4 is given in section 4 .

A hypergraph $H$ is a $(d, r)$-regular if every hypervertex is incident to $d$ hyperedges, and every hyperedge contains $r$ hypervertices. If $H$ is a $(d, r)$-regular hypergraph, then the associated bipartite graph $B_{H}$ is $(d, r)$-semiregular. Let $V_{1}=V(H), V_{2}=E(H)$ and $d \geq r$. Set $n=\left|V_{1}\right|$ and $m=\left|V_{2}\right|$. Then we have $\mathbf{A}^{[1]}=\mathbf{A}(H)$ and $\mathbf{A}^{[2]}=\mathbf{A}\left(H^{*}\right)$. By Theorems 3 and 4, we obtain the following result. Let $\operatorname{Spec}(\mathbf{B})$ be the set of all eigenvalues of the square matrix $\mathbf{B}$.

Theorem 5 Let $H$ be a finite, connected $(d, r)$-regular hypergraph with $d \geq r$. Set $n=|V(H)|$ and $m=|E(H)|$. Then

$$
\begin{aligned}
& \zeta(H, u, t)^{-1}=\left(1-(1-u)^{2} t\right)^{\epsilon-\nu}(1+(1-u)(r-1+u) t)^{m-n} \\
\times & \prod_{j=1}^{n}\left(1-\left(\lambda_{j}^{2}-(1-u)(d+r-2+2 u)\right) t+(1-u)^{2}(d-1+u)(r-1+u) t^{2}\right) \\
= & \left(1-(1-u)^{2} t\right)^{\epsilon-\nu}(1+(1-u)(r-1+u) t)^{m-n} \operatorname{det}\left(\mathbf{I}_{n}-(\mathbf{A}(H)-(r-2\right. \\
& \left.\left.\left.+(d+r-4) u+2 u^{2}\right) \mathbf{I}_{n}\right) t+(1-u)^{2}(d-1+u)(r-1+u) t^{2} \mathbf{I}_{n}\right) \\
= & \left(1-(1-u)^{2} t\right)^{\epsilon-\nu}(1+(1-u)(d-1+u) t)^{n-m} \operatorname{det}\left(\mathbf{I}_{m}-\left(\mathbf{A}\left(H^{*}\right)-(d-2\right.\right. \\
& \left.\left.\left.+(d+r-4) u+2 u^{2}\right) \mathbf{I}_{m}\right) t+(1-u)^{2}(d-1+u)(r-1+u) t^{2} \mathbf{I}_{m}\right),
\end{aligned}
$$

where $\epsilon=n d=m r, \nu=n+m$ and $\operatorname{Spec}(\mathbf{A}(H))=\left\{ \pm \lambda_{1}, \cdots, \pm \lambda_{n}, 0, \cdots, 0\right\}$.

In the case of $u=0$, we obtain Theorem 16 in [8]. 
Corollary 2 (Storm) Let $H$ be a finite, connected $(d, r)$-regular hypergraph with $d \geq r$. Set $n=|V(H)|, m=|E(H)|$ and $q=(d-1)(r-1)$. Then

$$
\begin{aligned}
\zeta_{H}(t)^{-1} & =(1-t)^{\epsilon-\nu}(1+(r-1) t)^{m-n} \operatorname{det}\left(\mathbf{I}_{n}-(\mathbf{A}(H)-r+2) t+q t^{2}\right) \\
& =(1-t)^{\epsilon-\nu}(1+(d-1) t)^{n-m} \operatorname{det}\left(\mathbf{I}_{m}-\left(\mathbf{A}\left(H^{*}\right)-d+2\right) t+q t^{2}\right),
\end{aligned}
$$

where $\epsilon=n d=m r$ and $\nu=n+m$.

\section{A proof of Theorem 4}

The argument is an analogue of Hashimoto's method [4].

By Theorem 1, we have

$$
\zeta(G, u, t)^{-1}=\left(1-(1-u)^{2} t^{2}\right)^{\epsilon-\nu} \operatorname{det}\left(\mathbf{I}_{\nu}-t \mathbf{A}+(1-u) t^{2}\left(\mathbf{Q}_{G}+u \mathbf{I}_{\nu}\right)\right) .
$$

Let $V_{1}=\left\{u_{1}, \cdots, u_{n}\right\}$ and $V_{2}=\left\{v_{1}, \cdots, v_{m}\right\}$. Arrange vertices of $G$ in $n+m$ blocks: $u_{1}, \cdots, u_{n} ; v_{1}, \cdots, v_{m}$. We consider the matrix $\mathbf{A}=\mathbf{A}(G)$ under this order. Then, let

$$
\mathbf{A}=\left[\begin{array}{cc}
\mathbf{0} & \mathbf{E} \\
{ }^{t} \mathbf{E} & \mathbf{0}
\end{array}\right]
$$

where ${ }^{t} \mathbf{E}$ is the transpose of $\mathbf{E}$.

Since $\mathbf{A}$ is symmetric, there exists a orthogonal matrix $\mathbf{W} \in O(m)$ such that

$$
\mathbf{E W}=\left[\begin{array}{ll}
\mathbf{F} & \mathbf{0}
\end{array}\right]=\left[\begin{array}{cccccc}
\mu_{1} & & 0 & 0 & \cdots & 0 \\
& \ddots & & \vdots & & \vdots \\
\star & & \mu_{n} & 0 & \cdots & 0
\end{array}\right] \text {. }
$$

Now, let

$$
\mathbf{P}=\left[\begin{array}{cc}
\mathbf{I}_{n} & \mathbf{0} \\
\mathbf{0} & \mathbf{W}
\end{array}\right]
$$

Then we have

$$
{ }^{t} \mathbf{P A P}=\left[\begin{array}{ccc}
\mathbf{0} & \mathbf{F} & \mathbf{0} \\
{ }^{t} \mathbf{F} & \mathbf{0} & \mathbf{0} \\
\mathbf{0} & \mathbf{0} & \mathbf{0}
\end{array}\right]
$$

Furthermore, we have

$$
{ }^{t} \mathbf{P}\left(\mathbf{Q}_{G}+u \mathbf{I}_{\nu}\right) \mathbf{P}=\mathbf{Q}_{G}+u \mathbf{I}_{\nu} .
$$

Thus,

$$
\begin{aligned}
& \zeta(G, u, t)^{-1}=\left(1-(1-u)^{2} t^{2}\right)^{\epsilon-\nu}\left(1+(1-u)\left(q_{2}+u\right) t^{2}\right)^{m-n} \operatorname{det}\left[\begin{array}{cc}
a \mathbf{I}_{n} & -t \mathbf{F} \\
-t^{t} \mathbf{F} & b \mathbf{I}_{n}
\end{array}\right] \\
= & \left(1-(1-u)^{2} t^{2}\right)^{\epsilon-\nu}\left(1+(1-u)\left(q_{2}+u\right) t^{2}\right)^{m-n} \operatorname{det}\left[\begin{array}{cc}
a \mathbf{I}_{n} & \mathbf{0} \\
-t^{t} \mathbf{F} & b \mathbf{I}_{n}-a^{-1} t^{2}{ }^{t} \mathbf{F F}
\end{array}\right] \\
= & \left(1-(1-u)^{2} t^{2}\right)^{\epsilon-\nu}\left(1+(1-u)\left(q_{2}+u\right) t^{2}\right)^{m-n} \operatorname{det}\left(a b \mathbf{I}_{n}-t^{2}{ }^{t} \mathbf{F} \mathbf{F}\right),
\end{aligned}
$$


where $a=1+(1-u)\left(q_{1}+u\right) t^{2}$ and $b=1+(1-u)\left(q_{2}+u\right) t^{2}$.

Since $\mathbf{A}$ is symmetric, ${ }^{t} \mathbf{F F}$ is symmetric and positive semi-definite, i.e., the eigenvalues of ${ }^{t} \mathbf{F F}$ are of form:

$$
\lambda_{1}^{2}, \cdots, \lambda_{n}^{2}\left(\lambda_{1}, \cdots, \lambda_{n} \geq 0\right)
$$

Therefore it follows that

$$
\zeta(G, u, t)^{-1}=\left(1-(1-u)^{2} t^{2}\right)^{\epsilon-\nu}\left(1+(1-u)\left(q_{2}+u\right) t^{2}\right)^{m-n} \prod_{j=1}^{n}\left(a b-\lambda_{j}^{2} t^{2}\right) .
$$

But, we have

$$
\operatorname{det}(\lambda \mathbf{I}-\mathbf{A})=\lambda^{m-n} \operatorname{det}\left(\lambda^{2} \mathbf{I}-{ }^{t} \mathbf{F F}\right),
$$

and so

$$
\operatorname{Spec}(\mathbf{A})=\left\{ \pm \lambda_{1}, \cdots, \pm \lambda_{n}, 0, \cdots, 0\right\} .
$$

Thus, there exists a orthogonal matrix $\mathbf{S}$ such that

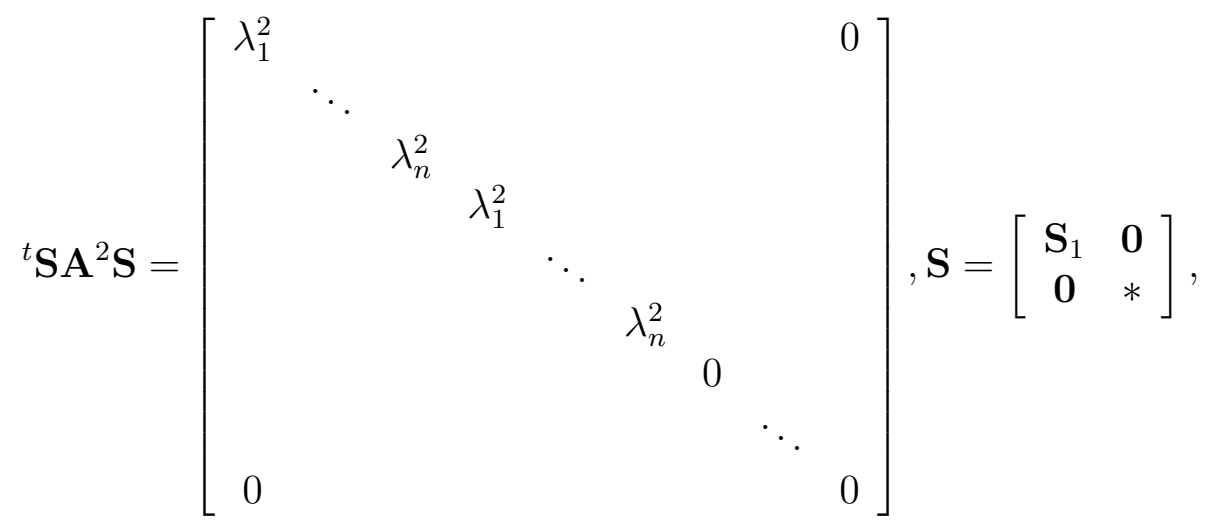

where $\mathbf{S}_{1}$ is an $n \times n$ matrix. Furthermore, we have

$$
\mathbf{A}^{2}=\mathbf{A}_{2}+\left(\mathbf{Q}_{G}+\mathbf{I}_{\nu}\right)
$$

where $\mathbf{A}_{2}=\left(\left(\mathbf{A}_{2}\right)_{u v}\right)_{u, v \in V(G)}$ :

$$
\left(\mathbf{A}_{2}\right)_{u v}=\text { the number of reduced }(u, v)-\text { paths with length } 2 \text {. }
$$

By the definition of the graphs $G^{[i]}(i=1,2)$,

$$
\mathbf{A}^{2}=\left[\begin{array}{cc}
\mathbf{A}^{[1]}+\left(q_{1}+1\right) \mathbf{I}_{n} & \mathbf{0} \\
\mathbf{0} & \mathbf{A}^{[2]}+\left(q_{2}+1\right) \mathbf{I}_{m}
\end{array}\right] .
$$

Thus,

$$
{ }^{t} \mathbf{S A}^{2} \mathbf{S}=\left[\begin{array}{cc}
\mathbf{S}_{1}^{-1} \mathbf{A}^{[1]} \mathbf{S}_{1}+\left(q_{1}+1\right) \mathbf{I}_{n} & \mathbf{0} \\
\mathbf{0} & *
\end{array}\right]
$$

Therefore, it follows that

$$
\mathbf{S}_{1}^{-1} \mathbf{A}^{[1]} \mathbf{S}_{1}=\left[\begin{array}{ccc}
\lambda_{1}^{2}-\left(q_{1}+1\right) & & 0 \\
& \ddots & \\
0 & & \lambda_{n}^{2}-\left(q_{1}+1\right)
\end{array}\right] .
$$


Hence

$$
\operatorname{det}\left(a b \mathbf{I}_{n}-\left(\mathbf{A}^{[1]}+\left(q_{1}+1\right) \mathbf{I}_{n}\right) t^{2}\right)=\prod_{j=1}^{n}\left(a b-\lambda_{j}^{2} t^{2}\right) .
$$

Thus, the second equation follows.

Similarly to the proof of the second equation, the third equation is obtained.

\section{Acknowledgment}

This work is supported by Grant-in-Aid for Science Research (C) in Japan. We would like to thank the referee for valuable comments and helpful suggestions.

\section{References}

[1] L. Bartholdi, Counting paths in graphs, Enseign. Math. 45 (1999), 83-131.

[2] H. Bass, The Ihara-Selberg zeta function of a tree lattice, Internat. J. Math. 3 (1992) 717-797.

[3] D. Foata and D. Zeilberger, A combinatorial proof of Bass's evaluations of the IharaSelberg zeta function for graphs, Trans. Amer. Math. Soc. 351 (1999), 2257-2274.

[4] K. Hashimoto, Zeta Functions of Finite Graphs and Representations of $p$-Adic Groups, Adv. Stud. Pure Math. Vol. 15, Academic Press, New York, 1989, pp. 211280.

[5] Y. Ihara, On discrete subgroups of the two by two projective linear group over $p$-adic fields, J. Math. Soc. Japan 18 (1966) 219-235.

[6] M. Kotani and T. Sunada, Jacobian tori associated with a finite graph and its abelian covering graph, Adv. in Appl. Math. 24 (2000) 89-110.

[7] H. M. Stark and A. A. Terras, Zeta functions of finite graphs and coverings, Adv. Math. 121 (1996), 124-165.

[8] C. K. Storm, The zeta function of a hypergraph, preprint.

[9] T. Sunada, L-Functions in Geometry and Some Applications, in Lecture Notes in Math., Vol. 1201, Springer-Verlag, New York, 1986, pp. 266-284.

[10] T. Sunada, Fundamental Groups and Laplacians (in Japanese), Kinokuniya, Tokyo, 1988. 